\title{
Exosomal circular RNA sorting mechanisms and their function in promoting or inhibiting cancer (Review)
}

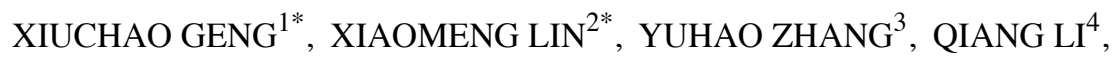 \\ YAJING GUO ${ }^{5}$, CHUAN FANG ${ }^{3}$ and HONG WANG ${ }^{1}$
}

\begin{abstract}
${ }^{1}$ Faculty of Integrated Traditional Chinese and Western Medicine, Hebei University of Chinese Medicine, Shijiazhuang, Hebei 050091; Departments of ${ }^{2}$ Breast Surgery and ${ }^{3}$ Neurosurgery, Affiliated Hospital of Hebei University, Baoding, Hebei 071000; ${ }^{4}$ Faculty of Acupuncture-Moxibustion and Tuina, Hebei University of Chinese Medicine, Shijiazhuang, Hebei 050200; ${ }^{5}$ Scientific Research Center, Hebei University of Chinese Medicine, Shijiazhuang, Hebei 050091, P.R. China
\end{abstract}

Received September 12, 2019; Accepted February 13, 2020

DOI: $10.3892 / \mathrm{ol} .2020 .11449$

\begin{abstract}
Exosomes are nanoscale phospholipid bilayer vesicles that can be artificially engineered into vectors for the treatment of cancer. Circular RNA (circRNA), a type of non-coding RNA, has crucial regulatory functions in various aspects of cancer, such as tumorigenesis, apoptosis, proliferation, invasion, metastasis and chemo- and radiotherapeutic resistance, as well as in cancer prognosis. Notably, the exosomal transfer of circRNAs may function to both promote and inhibit cancer. Numerous studies have addressed the importance of circRNAs in cancer and non-coding RNAs (such as microRNAs and long non-coding RNAs) in exosomes. However, little research has focussed on a class of RNAs called exosomal circRNAs. The present review discusses current studies regarding exosomal circRNAs, including their biogenesis and biological functions, their abundance in exosomes
\end{abstract}

Correspondence to: Professor Hong Wang, Faculty of Integrated Traditional Chinese and Western Medicine, Hebei University of Chinese Medicine, 326 Xinshi South Road, Shijiazhuang, Hebei 050091, P.R. China

E-mail: bossw@vip.sina.com

Professor Chuan Fang, Department of Neurosurgery, Affiliated Hospital of Hebei University, 212 Yuhua East Road, Baoding, Hebei 071000, P.R. China

E-mail: chuanfang@hbu.edu.cn

*Contributed equally

Abbreviations: circRNAs, circular RNAs; CRC, colorectal cancer; $\mathrm{EV}$, extracellular vesicle; GC, gastric cancer; HCC, hepatocellular carcinoma; lncRNAs, long non-coding RNAs; miRNAs, microRNAs; mRNA, messenger RNA; MVBs, multivesicular bodies; ncRNA, non-coding RNA; PC, pancreatic cancer; PDAC, pancreatic ductal adenocarcinoma; shRNAs, short hairpin RNAs; siRNAs, small interfering RNAs; tRNA, transfer RNA; UCB, urothelial carcinoma

Key words: exosomes, circRNAs, exosomal circRNAs, cancer, mechanism, function and possible sorting mechanisms and their potential roles in both promoting and inhibiting cancer. It is predicted that in the next five years there will be increasing research exploring the functional mechanisms of exosomal circRNA in various diseases, in particular their roles in cancer genesis and progression.

\section{Contents}

1. Introduction

2. Biogenesis of circRNAs

3. Functions of circRNAs

4. Exosomal circRNAs and their sorting mechanisms

5. Exosomal circRNAs as tumour biomarkers

6. Exosomes containing circRNAs function in promoting or inhibiting cancer

7. Conclusion

\section{Introduction}

Exosomes, ranging from 30-150 nm in diameter, are nanoscale extracellular phospholipid bilayer vesicles, which originate from endosomes and are stored within multivesicular bodies (MVBs) (1). When MVBs fuse with the cytomembrane, exosomes are released into the extracellular environment or fuse with target cells, resulting in a series of phenotypic changes $(2,3)$. Exosomes encapsulate and transfer a myriad of functional molecular cargoes, including proteins (4), lipids, metabolites, DNA, messenger RNA (mRNA), ribosomal RNA, microRNA (miRNA), transfer RNA (tRNA), long non-coding RNA (lncRNA) and circular RNA (circRNA) $(5,6)$. As exosomes are released into the external environment, circRNA begins cycling and binds miRNA or proteins to exert various biological functions. Numerous studies have confirmed that exosomes act as crucial mediators of intercellular communication (7-9). In addition, they function in numerous physiological processes, such as blood coagulation (10), tissue repair, skin regeneration (11) and immune modulation (12); therefore, exosomes play roles in cancer and other pathological processes such as in cancer, cerebral ischemia and obesity (13-15). 
circRNA belongs to the endogenous non-coding RNA (ncRNA) family. Unlike linear RNA, circRNA is a closed circular molecule that has a covalently closed loop structure, lacking a poly A tail or 5'-3' polarity (16). Although circRNA was initially considered as a non-functional by-product of aberrant RNA splicing, numerous studies have investigated this molecule class (17-19), and the development of bioinformatics approaches and next-generation deep sequencing technology have contributed to the discovery and identification of an increasing number of circRNAs with regulatory functions (19). circRNAs are molecules that display high enrichment and relative stability, diversity and evolutionary conservation; they also exhibit tissue-specific and developmental phase-specific expression (20). These characteristics indicate that circRNAs have distinct properties and diverse cellular regulatory functions, including the regulation of cellular processes, such as proliferation and apoptosis (21-23). Furthermore, it has been revealed that circRNAs have crucial regulatory functions in various aspects of cancer, such as tumorigenesis (24), proliferation, migration, invasion (25), metastasis (26), apoptosis, and chemotherapy and radiation resistance $(27,28)$, as well as in cancer prognosis (29).

Previous studies have demonstrated that circRNAs are stable in cells and within exosomes (6). It has been revealed that exosomal circRNAs may have important regulatory functions, and due to their unique structure and high specificity, the use of combinations of exosomes and circRNAs may increase the potential clinical applications of these molecules as markers in the diagnosis and prognosis of cancer $(30,31)$. For example, high expression of plasma exosomal circ-PDE8A is associated with lymphatic invasion and advanced tumour stage, as well as poor survival in patients with pancreatic ductal adenocarcinoma (PDAC) (32). Therefore, exosomal circ-PDE8A may be used as a marker for determining diagnosis or progression of the disease in patients with PDAC (32). Notably, regarding the role of exosomal circRNA in cancer, exosomes containing circRNAs may function to promote or inhibit cancer progression. Exosomal circRNAs promote the progression (proliferation and invasiveness) of cancer, the generation of premetastatic niches and the occurrence of metastasis $(33,34)$. By contrast, exosomal circRNAs also regulate tumour immunity and immunotherapy and play a role in cancer treatment $(35,36)$.

In previous years, numerous studies on exosomal lncRNAs and miRNAs in cancer have been conducted, but relatively little attention has been devoted to exosomal circRNAs. In the current review, an overview of exosomal circRNAs is provided; the biogenesis and biological functions of circRNAs are discussed, the abundance of circRNAs in exosomes and their possible sorting mechanisms are analysed and their potential emerging roles in promoting or inhibiting cancer progression are examined.

\section{Biogenesis of circRNAs}

Unlike linear RNAs, circRNAs are generated by back-splicing or exon skipping of pre-mRNAs, with direct back-splicing occurring more frequently than exon skipping (37). circRNAs are cyclized into a continuous closed-loop structure that lacks a polyadenylation (poly A) tail and 5' cap (38). Based on their composition, circRNAs are divided into four categories: Exonic circRNA (ecircRNAs), exon-intron circRNAs (EIciRNAs), circular intronic RNAs (ciRNAs) and tRNA intronic circular RNAs (tricRNAs). Currently, three mechanisms for circRNA biogenesis are widely accepted (Fig. 1): RNA-binding protein (RBP)-mediated circularization, intron pairing-driven circularization and lariat-driven circularization. First, the RBP-mediated and intron pairing-driven circularization mechanisms of circRNA biogenesis occur via the direct back-splicing pathway (18). RBPs have an important function in promoting circRNA biogenesis by regulating adjacent splice sites (Fig. 1A). For instance, the splicing factors Muscleblind (39), Quaking (40), adenosine deaminase RNA specific (41) and DExH-box helicase 9 (42) are all reported to participate in the formation of circRNA. Furthermore, circularization can also occur by complementary pairing of flanking introns that contain inverted complementary sequences (Fig. 1B) (43). Finally, lariat-driven circularization is facilitated by an exon-skipping event (Fig. 1C). Internal splicing facilitates the removal of the flanking intronic sequence, allowing the production of ecircRNAs (44); if these flanking sequences are retained, the constructs are called EIciRNAs (45). Additionally, ciRNAs are generated via lariat-driven circularization, which is facilitated by a ciRNA-specific consensus motif (Fig. 1D) (46). TricRNAs are generated by a combination of the released intron terminal ends, which come from spliced pre-tRNAs via the tRNA splicing endonuclease complex (Fig. 1E) (47).

\section{Functions of circRNAs}

circRNAs are abundant in the cytoplasm, evolutionarily conserved among species and relatively stable compared with their linear counterparts (20). These features provide circRNAs with a number of potential functions. The main functions of circRNAs are regulating the expression of parental genes, acting as miRNA or RBP sponges and being translated into peptides or proteins.

EIciRNAs interact with U1 small nuclear ribonucleoproteins, and they increase host gene transcription by binding to RNA polymerase II (RNA pol II) (45). Certain ciRNAs and the RNA pol II complex directly interact to regulate parental gene transcription (Fig. 2A) (46). circRNAs also function as miRNA sponges (Fig. 2B). These circRNAs contain miRNA response elements, which facilitate the binding of circRNAs with miRNAs, thereby sequestering the miRNAs away from target mRNA molecules (48). For example, circ_0001730 is upregulated in glioblastoma and promotes glioblastoma cell proliferation and invasion by serving as a ceRNA for miR-326 to regulate the $\mathrm{Wnt} 7 \mathrm{~B} / \beta$-catenin pathway (49). Moreover, certain circRNAs are rich in binding sites for RBPs, so they inhibit the biological function of RBPs by functioning as protein sponges (Fig. 2C) (50). For instance, circ-Sirt1 interacts with NF- $\mathrm{KB}$ p65 in the cytoplasm, thereby sequestering the nuclear translocation of p65 (induced by inflammation), and inhibiting the expression of inflammatory factors (51). circRNAs are considered to serve as protein scaffolds by harbouring binding sites for the assembly of two or more proteins, such as enzymes and their substrates, which may subsequently form large protein complexes (37). For example, circ-forkhead 
A

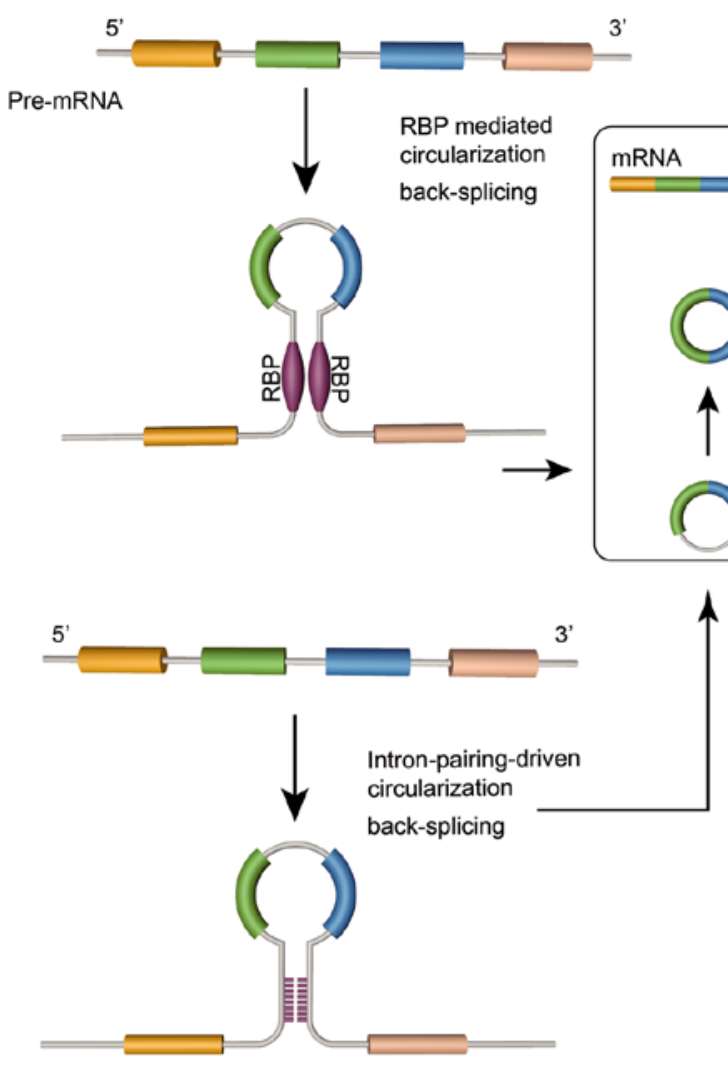

B

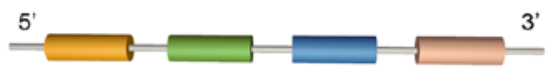$$
c
$$

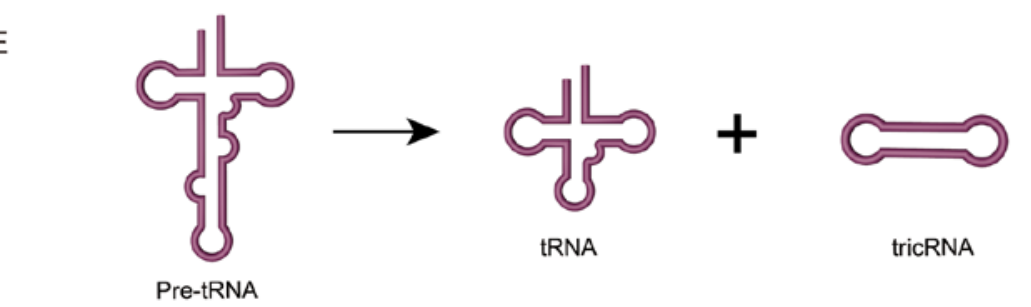

Figure 1. Mechanisms for circRNA biogenesis. (A) RBP-mediated circularization. (B) Lariat-driven circularization. (C) Intron pairing-driven circularization. (D) ciRNAs are also generated via lariat-driven circularization. (E) Biogenesis of tricRNAs. circRNA, circular RNA; RBP, RNA-binding protein; ciRNA, circular intronic RNA; tricRNA, tRNA intronic circular RNA; ecircRNA, exonic circRNA; EIciRNA, exon-intron circRNA; tRNA, transfer RNA.

A

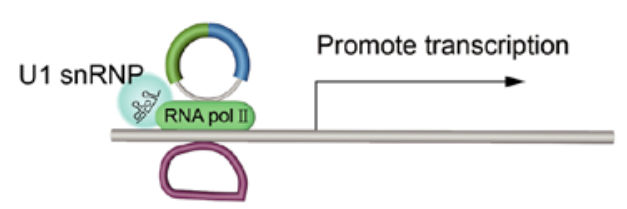

C

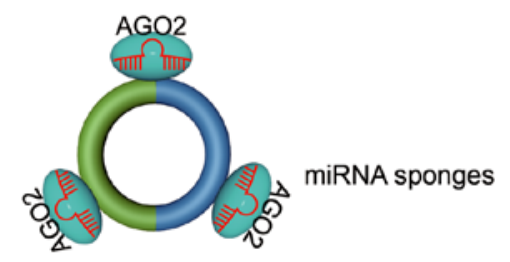

B

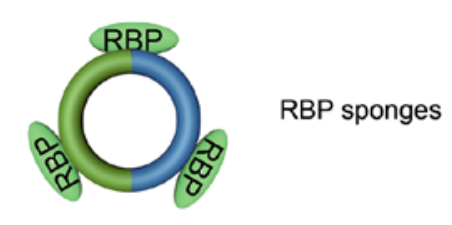

D
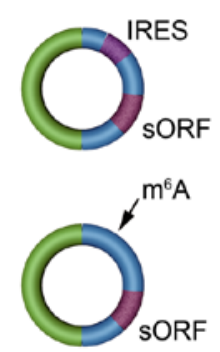

ceececuccumamm

Protein

Figure 2. Potential biological functions of circRNAs. (A) EIciRNAs and ciRNAs regulate parental gene transcription. (B) circRNAs function as protein sponges. (C) circRNAs function as miRNA sponges. (D) Certain circRNAs with an IRES or m6A-driven sORF can be translated. circRNA, circular RNA; EIciRNA, exon-intron circRNA; ciRNA, circular intronic RNA; IRES, internal ribosome entry site; sORF, small open reading frame; U1 snRNP, U1 small nuclear ribonucleoproteins; RNA pol II, RNA polymerase II; RBP, RNA-binding protein; AGO2, Argonaute 2; miRNA, microRNA. 
box O3 (Foxo3) may generate a circ-Foxo3-p21-CDK2 ternary complex by interacting with $\mathrm{CDK} 2$ and $\mathrm{p} 21$, which functions to inhibit CDK2 (23). Certain circRNAs, such as circ-SNF 2 histone linker PHD RING helicase (SHPRH), which has an internal ribosome entry site (IRES)-driven small open reading frame (sORF) for the translation of SHPRH-146aa, is a tumour suppressor in glioma (52). Moreover, it was revealed that IRESs and the N6-methyladenosine modification may drive sORFs for the translation of circRNAs (Fig. 2D) (53).

\section{Exosomal circRNAs and their sorting mechanisms}

circRNAs are abundant in exosomes. Exosomes containing mRNA and miRNA were first reported by Valadi et al (54). Numerous studies have since demonstrated the existence of various small ncRNA species (in addition to miRNA, lncRNA and mRNA) in exosomes (55-58). Subsequently, Li et al (6) reported the existence of a large quantity of circRNAs in exosomes. Furthermore, it has been observed that circRNAs are highly enriched and stable in exosomes, particularly in tumour-derived exosomes, compared with cells which secrete circRNA. Notably, circRNAs are incorporated into exosomes more frequently than their linear counterparts (59). In theory, cells can equally load circRNAs and the linear forms of the same mRNAs into extracellular vesicles (EVs). Therefore, the reasons for this difference, and the reason that circRNAs are more abundant in exosomes compared with in their producer cells, can be explained by three possible mechanisms. Primarily, unlike linear RNAs, circRNAs have a relatively long half-life because they are covalently closed loops lacking poly A tails or 5'-3' ends; thus, it is hypothesized that circRNAs are resistant to exonucleolytic degradation $(38,48)$. Linear RNAs and circRNAs exhibit markedly different production rates and stabilities, with the production and degradation rates of linear RNAs being significantly faster compared with circRNA (60). Thus, circRNAs are more stable compared with linear transcripts in cells, with the half-life of the majority of circRNA species exceeding $48 \mathrm{~h}$, compared with $<20 \mathrm{~h}$ in the linear RNA molecules (61). Thus, despite the low generation efficiency of circRNAs, their transcripts can accumulate at higher levels compared with linear mRNAs due to their long half-lives. Notably, circRNAs have been demonstrated to accumulate in cells with slow division rates, ultimately reaching relatively high levels compared with linear RNAs (62). Secondly, EVs may be used as a mechanism for circRNA clearance in cells. Cells can remove cytoplasmic circRNAs by releasing cargo-bearing EVs, such as microvesicles and exosomes. By releasing circRNAs from cells in EVs, they are removed from the cell via export into the extracellular space (63); this may explain why circRNAs are abundant in exosomes and are potential biomarkers and therapeutic targets during disease processes (35). Finally, exosomal circRNA is highly abundant and stable, perhaps due to the protective effects of exosomes or certain sequence features, as well as its protein partners. Generally, exosomes safely prevent cargo from being further cleared or undergoing RNA damage or degradation, as their membrane is double-layered and they are nanosized, which helps to prolong the circulation half-life and enhance the biological activities of circRNAs (64).
In summary, the aforementioned reasons may explain why exosomes have more abundant levels of specific circRNAs compared with producer cells, and why the expression of circRNAs is higher compared with corresponding linear RNAs.

Possible mechanisms by which exosomes select RNA cargo. Exosomes contain numerous different varieties of bioactive cargoes derived from cells, and these cargoes are heterogeneous, reflecting the type and state of their cells of origin (65). However, exosomes with different origins may contain common cargoes, and cargoes in exosomes derived from the same cell may differ. Hence, exosomes contain specific cellular RNA subsets that are distinct or tissue-specific. The detection of exosomal RNAs by transcript-specific reverse transcription-quantitative PCR or high-throughput analyses revealed a difference between circRNA species in exosomes and the cytoplasm (6), which also reveals that circRNAs are actively, not passively, incorporated into exosomes and that this sorting process is selective (Fig. 3) (66). Despite having a substantial understanding of cargo molecule transport from cells to exosomes, knowledge of the basic mechanisms underlying cargo selection remains limited.

A number of defined RBPs can be used to recognize and sort RNAs with specific binding motifs into exosomes (67-69). These specific motifs are shared by RNAs in EVs, which may facilitate their targeting to EVs. For instance, RBP hnRNPA2B1 can bind and transfer miRNAs into exosomes via conserved sequences known as exosomes motifs, which are enriched in exosomes but not in cells (70). Moreover, RBP hnRNPA2B1 was reported to specifically regulate the inclusion of lncARSR into exosomes, and their specific binding depends on the sequence at the 5' end (58). However, the mechanism by which RBPs interact with the endosomal system is yet to be elucidated. Moreover, because the RNA-binding complex ESCRT-II serves a canonical role in MVB biogenesis (1), it may assist in selecting RNAs for incorporation into EVs. A study on circRNA sorting into exosomes indicated that this process was, at least partially, regulated by associated miRNA levels in the producer cells (6). Moreover, exosomal circRNAs are considered to retain biological activity and can abrogate the growth inhibition induced by miR-7 in recipient cells (6).

In summary, the complex mechanisms by which RNAs are selectively and specifically sorted into exosomes require further clarification. Several types of sorting mechanisms may be simultaneously active. Therefore, it is hypothesized that exosomal ncRNA sorting will become a future research hotspot.

\section{Exosomal circRNAs as tumour biomarkers}

The early diagnosis of cancer has long been a focus of research worldwide. In recent years, an increasing number of novel and accessible methods for cancer diagnosis have continued to emerge (71). Notably, exosomes can be detected in nearly all types of human bodily fluids, such as blood (72), breast milk (73), bile, saliva (74), tears, urine (75), semen (76), ascites, synovial fluid, cerebrospinal fluid (77), amniotic fluid (78), bronchoalveolar lavage fluid (79) and faeces (Fig. 4A). Compared with biomarkers in tumour tissues, which can only 


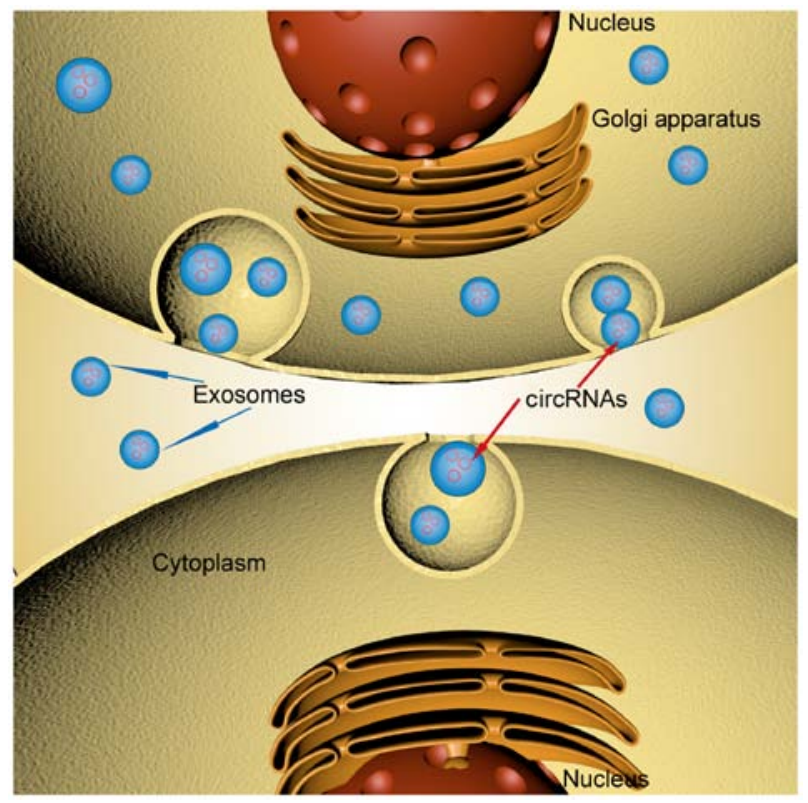

Figure 3. Exosomes are abundant in specific circular RNAs and facilitate intercellular communication. circRNA, circular RNA.

be obtained by biopsy, exosomal biomarkers can be detected using samples that are easier and less invasive to obtain. This is an advantage of using exosomes as a biomarker. As an exosomal cargo, circRNAs themselves have several notable characteristics, including high abundance and diversity, stability, conservation and localization and expression specificity (18). In recent years, numerous studies have explored the advantages of exosomal circRNAs. These features indicate that circRNAs may also be suitable as potential diagnostic biomarkers for cancer $(19,23)$ or other diseases such as coronary artery disease (80), Alzheimer's disease (81) and systemic lupus erythematosus (82). For instance, high expression of blood exosomal circ-PDE8A was revealed to be associated with lymphatic invasion and tumour stage, as well as poor survival in patients with PDAC. Therefore, this exosomal circRNA may be used as a marker for determining diagnosis or progression in patients with PDAC (32). In another study, it was observed that exosomes from pancreatic cancer overexpressed circ-IRAS (34). Circ-IARS could enter HUVECs via exosomes and promote tumor invasion and metastasis (34). Exosomal circRNA_100284 was reported to induce cell cycle acceleration and promote cell proliferation via miR-217, which targets enhancer of zeste homologue 2 (EZH2) in cancers (83). Exosomal circRNA_100284 may also serve as a biomarker for arsenite exposure (83). Recently, researchers demonstrated that human plasma is rich in mRNA, circRNA and lncRNA via EV long RNA sequencing (exLR-Seq), and these RNAs may be useful as biomarkers for tumour diagnosis and prognosis. The aforementioned study reported that 8 exLRs may be used as diagnostic biomarkers, having utility beyond the current diagnostic approaches (such as $\alpha$-feto protein) for hepatocellular carcinoma ( $\mathrm{HCC}$ ), with high sensitivity and specificity (84). These findings have crucial functional and clinical implications.

In summary, exosomal circRNAs are clinically valuable as a new generation of biomarkers for the early diagnosis and prognosis of various diseases, particularly cancer, as well as the evaluation of therapeutic effects (12).

\section{Exosomes containing circRNAs function in promoting or inhibiting cancer progression}

Function of exosomal circRNAs. Several studies have reported that circRNAs can function in cancer by either promoting or inhibiting tumour progression and regulating the biological behaviour of malignant tumours, including cell proliferation, migration, invasion and metastasis $(26,85,86)$. However, circRNAs in exosomes are still rarely studied. Notably, exosomal circRNAs appear to play a pleiotropic role in cancer due to their involvement in intercellular communication $(22,87)$. On the one hand, exosomes containing circRNAs promote the progression of cancer, the generation of premetastatic niches and the occurrence of metastasis $(33,34)$. On the other hand, exosomes may potentially function in tumour immune regulation and cancer therapy $(35,36)$.

Exosomal circRNAs promote cancer progression. circRNAs in exosomes derived from patients with multiple tumours are distinct from those of healthy individuals (88), indicating that they may have great clinical significances and research value. A number of studies have investigated the mechanism by which exosomal circRNAs function in cancer development. A study revealed that the exosomal circ-PDE8A is released by tumours facilitates invasive development in a miR-338-MET transcriptional regulator MACC1-MET proto-oncogene receptor tyrosine kinase pathway-dependent manner in pancreatic cancer (PC), revealing that circ-PDE8A enhances tumour invasiveness via the communication mediated by exosomes (32). Another study reported that exosomal circRNA_100284 accelerated the cell division cycle and promoted proliferation, via sponging miR-217. Overexpression of circRNA_100284 enhanced the invasion and migration of, and increased the formation of tumour colonies, by stimulating the downstream signalling pathway and increasing the expression of EZH2 and cyclin-D1 in human hepatic cells (83). Furthermore, this circRNA also affects the malignant transformation of cells (83). In another study, gastric cancer (GC) cell-derived exosomes transferred ciRS-133 into preadipocytes, which facilitated preadipocyte differentiation into brown-like cells via the activation of PR/SET domain 16 and the inhibition of miR-133 (8). Additionally, this type of exosome can participate in white adipose tissue browning and affect cancer cachexia (8), a syndrome characterized by weight loss due to muscle and/or fat loss. Cancer cachexia results in functional impairment, decreased physical ability and it is associated with a poor prognosis (89). The same mechanisms have been reported in other studies $(90,91)$. It can be concluded from the aforementioned studies that exosomes can directly transfer circRNAs from cancer cells to surrounding cells, and then further mediate the biological functions of recipient cells. Meanwhile, circRNAs serve as competing endogenous RNAs or miRNA sponges to promote the progression of multiple cancer types.

In summary, exosomal circRNAs influence the promotion of malignant transformation, proliferation, invasiveness and migration in cancer, and are associated with cancer cachexia. 


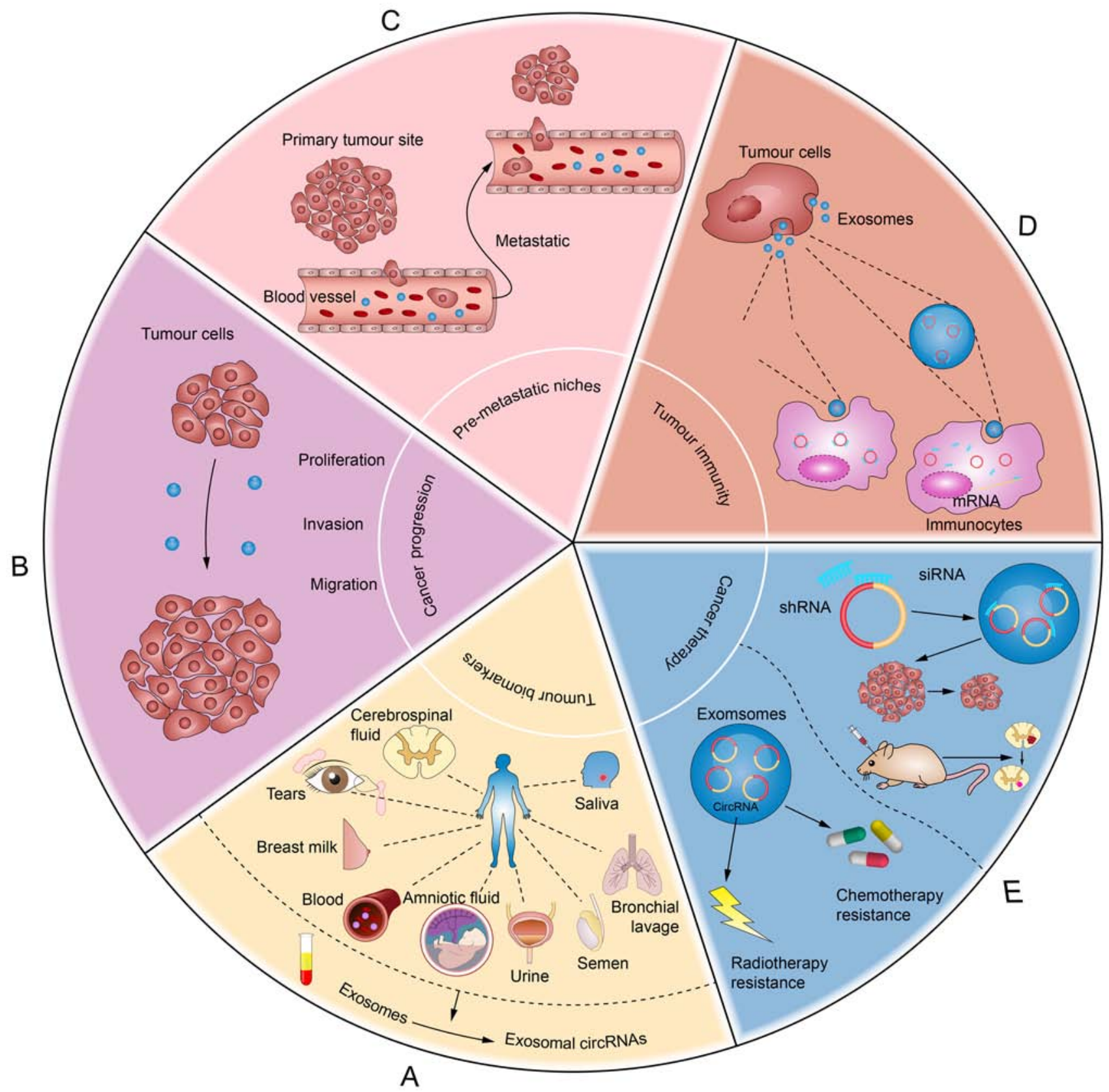

Figure 4. Exosomal circRNAs function in promoting or inhibiting cancer. (A) Exosomes are secreted by various cell types and can be detected in nearly all types of human bodily fluids and secretions, such as blood, breast milk, bile, saliva, tears, urine, semen, ascites, synovial fluid, cerebrospinal fluid, amniotic fluid, bronchoalveolar lavage fluid and faeces. (B) Exosomal circRNAs are involved in promoting the malignant transformation, proliferation, invasion and migration of cancer. (C) Exosomal circRNAs have pivotal functions in the generation of premetastatic niches and tumour metastasis. (D) Exosomal circRNAs regulate tumour immunity and immunotherapy. (E) Functions of exosomal circRNAs in cancer therapy. circRNA, circular RNA; shRNA, short hairpin RNA; siRNA, short interfering RNA.

Notably, acting as miRNA sponges is the most common mechanism. Further investigation is needed to explore other exosomal circRNAs that serve a role in promoting cancer progression (Fig. 4B).

Exosomal circRNAs promote the generation of premetastatic niches and tumour metastasis. Exosomes are vital in mediating cell-to-cell communication and delivering cargo from donor cells to recipient cells, regardless of whether the recipient cells are located in a remote or nearby tissue, known as horizontal transfer (92). In cancer, exosomes are involved in the communication between tumour cells and the surrounding microenvironment (93). The interaction between malignant and surrounding tumour or normal cells can greatly affect tumour progression (94). Studies have reported that exosomal circRNAs have pivotal functions in premetastatic niche formation and metastasis (Fig. 4C) (12). For example, exosomes derived from cells with high metastatic potential and abundant circPTGR1 can potentially downregulate the interaction between miR-449a and MET in recipient cells. Thus, affecting cells with low metastatic potential and disrupting tumour microenvironment homeostasis, as well as promoting HCC progression (30). In addition, a previous study revealed that exosomal communication between GC cells aids 
in the transfer of circNRIP1. Exosomal circNRIP1 may sponge miR-149-5p to facilitate GC metastasis via the AKT1/mTOR signalling pathway (95). Pathological EMT is a key factor in the process of tumour progression and metastasis. Recently, a study observed that the level of circPRMT5 was increased in serum and urine exosomes from patients with UCB, and this was associated with cancer metastasis (96). CircPRMT5 may promote the EMT of UCB cells and result in an aggressive phenotype via sponging miR-30c.

Thus, exosomal circRNAs have a significant influence in promoting the generation of premetastatic niches and the occurrence of tumour metastasis.

Exosomal circRNAs may regulate tumour immunity and immunotherapy. Increasing studies have revealed that exosomes are closely associated with tumour immunity, resulting in either tumour suppression or tumour promotion (97-99). Whether they originate from the tumour or from immune cells determines the role of exosomes in the tumour immune response (100).

Exosomes from tumour cells have unique immunoregulatory effects on the immune system, and they can be used as a medium for regulating tumour cell-regulatory $\mathrm{T}$ cell (Treg) communication (55). For example, nasopharyngeal carcinoma (NPC)-exosome may induce expansion of the Treg population and enhance the suppressive functions of Tregs. The interactions of NPC-exosome and Tregs may be associated with the tumour microenvironment, allowing immune suppression as well as evading host immune surveillance (101). EVs derived from colorectal cancer (CRC) cells may induce phenotypic alteration of the $\mathrm{T}$ cells to Treg-like cells by activating transforming growth factor- $\beta /$ SMAD signalling and inactivating stress-activated protein kinase (SAPK) signalling 9 (102). Additionally, these Treg-like cells may promote tumour cell proliferation (102).

Accumulating evidence has revealed that exosomes and ncRNAs (miRNA and lncRNA) are critical to Treg cell homeostasis (103). For example, tumour exosomal miRNAs may induce immune tolerance and then exert an adverse immune effect (104). However, studies on exosomal circRNA regulation of Treg cells are relatively new, and further research is needed.

Tumour cell-derived exosomes may carry abnormally expressed circRNAs and shuttle them to surrounding cells (including immunocytes). circRNAs may become tumour antigens or bind miRNAs or proteins to regulate immunocompetence when reaching the target immunocytes. Furthermore, there are important interactions between circRNAs and miRNAs, as described above: circRNAs in exosomes may initially bind tumour-specific miRNAs or mRNAs via the circRNA-miRNA-mRNA axis, assisting in the shuttling of miRNAs between carcinoma cells and immunocytes, as well as enhancing their stability as exosomes transit the intercellular space (36). When exosomes fuse with the target immunocytes, they release their cargo, which perform notable functions in tumour immunity (Fig. 4D) (36). For example, miRNA-155 can be upregulated to potentiate immunotherapies of tumor-specific $\mathrm{CD}^{+} \mathrm{T}$ cells for cancer (105). Tumor-derived miR-214 targets tensin homolog (PTEN) resulting in an increase in Treg cell number and enhanced immune suppression (55). It is predicted that exosomal circRNAs are a promising target for tumour immunotherapy.

These findings indicate that malignant carcinoma cells and immunocytes exist continually in a dynamic equilibrium, in which exosomes serve a crucial role in maintaining homeostasis between these two cell types.

Exosomal circRNAs in cancer therapy. The primary prospects of exosome treatment include participating in intercellular information exchange and targeted drug delivery. As exosomes are nanoscale biological vesicles, their cargo is often protected so they have an innate advantage as a useful therapeutic vector (106). In addition, exosomes, as mediators of communication between cells, are highly safe, bioavailable and exhibit low systematic immunogenicity and toxicity compared with conventional targeting vectors (107). Exosomes have exhibited a more notable positive therapeutic effect on cancer compared with direct delivery of chemotherapy (107). Exosomes may also be used to solve a number of the issues surrounding currently used drug therapy methods, which involve programmable RNA, which has low uptake efficiency, is highly cytotoxic and is thus unsuitable for clinical practice (108). Researchers have proposed and verified the effectiveness of a new strategy for the generation of large amounts of RBC-derived EVs for delivering RNA drugs (109). The use of gold nanoparticle targeting approaches to generate specific types of exosomes offers an alternative for the selective, targeted elimination of cancer cells (110). In summary, exosomes are vesicles that can be artificially engineered into useful treatment vectors as a therapeutic agent against cancer $(13,109,111,112)$.

Regarding pharmaceutical cargo, exosomes can be loaded with chemotherapeutic drugs (113) for targeted therapy and immunoadjuvant-mediated immunotherapy (106), as well as ncRNAs and small interfering RNAs (siRNAs) (114). The transfer of siRNA can promote the exchange of genetic information and make a substantial difference in cellular biological behaviour. For example, nanovesicles that mimic exosomes can achieve targeted delivery of RNA interference chemotherapeutic drugs to malignant tumours, and evidence suggests that exosomes carrying siRNA may target c-Myc (115).

Exosomal delivery of circRNA would have a number of therapeutic advantages. Primarily, the control of natural circRNA expression in specific tissues and cells is likely to reduce adverse effects compared with synthetic molecular drugs. Moreover, one common phenomenon and major function of circRNA is their role as a miRNA sponge. Therefore, research on endogenous circRNA sponge structures may assist in the design and development of potent artificial sponges to regulate the function of miRNAs in disease. Additionally, the off-target effects of circRNAs may be low compared with siRNAs and miRNAs due to their short length. Indeed, off-target effects are a significant problem that limits the translation of small molecule RNAs into clinical practice (116). However, off-target effects do not negatively affect circRNA therapy, as circRNAs have a stable and specific structure (18). In addition, circRNA is not easily digested by ribonucleases and has a natural structure that makes it more stable than linear RNA. Due to the construction of engineered exogenous circRNAs, mRNA cyclization can effectively solve the issues facing linear mRNA, such as its shorter half-life, enabling it 
to permanently and efficiently express proteins in eukaryotic cells (117). In summary, it is likely that exosomal circRNA therapy will be a future gene therapy used for the treatment of various diseases.

Exosomes can also encapsulate both siRNAs and short hairpin RNAs (shRNAs) that are specifically designed to target and reduce the levels of specific circRNAs in tumour cells that have detrimental effects on patient outcomes (35). These siRNAs or shRNAs sponge miRNAs and promote the expression of antioncogenes that indirectly inhibit damage caused by circRNAs $(95,118)$. For example, three shRNAs that span the back-splicing site of the circRNA c transferrin receptor (cTFRC) were designed in a previous study to inhibit its expression. The results indicated that the downregulation of cTFRC suppresses bladder cancer cell invasion (118). Moreover, similar studies of circNRIP1 have been reported, and circNRIP1 silencing via circNRIP1 siRNA transfection notably inhibited the proliferation, invasion and migration of GC cells (95). This phenomenon was also observed in prostate cancer (119), CRC (120), PC (32), UCB (96), HCC (30,121) and glioblastoma $(122,123)$.

Therapeutic resistance is a major problem in the treatment of cancer. Notably, exosomal circRNAs were demonstrated to influence radioresistance in a study by Zhao et al (28), who used RNA-Seq to analyse the circRNAs present in EVs from U251 and radioresistant U251 cells. CircATP8B4 was demonstrated to promote glioma radioresistance by serving as a miR-766 sponge, and circATP8B4 in EVs may be a potential biomarker for glioma radioresistance. It was reported that exosome-transmitted miRNA may promote chemosensitivity in multiple cancer types such as colorectal cancer and head and neck cancer $(124,125)$, and recent studies have revealed that circRNAs mediate chemotherapy resistance in various types of cancer such as ovarian cancer and thyroid carcinoma (126-128). Hence, there is a novel hypothesis that exosomal circRNAs are influence chemotherapy resistance, but this still requires further experimental confirmation (Fig. 4E).

In summary, exosomes carrying various circRNAs may assist in promoting cancer progression. However, exosomes loaded with circRNA and engineered siRNAs that target specific circRNAs may be valuable in the development of drugs to inhibit tumour progression, and for accurate and effective therapy.

\section{Conclusion}

Exosomes encapsulate and transfer a myriad of functional molecular cargoes and serve as crucial mediators of intercellular communication. As an important cargo of exosomes, circRNAs have been demonstrated to serve crucial regulatory functions in various aspects of cancer, such as tumorigenesis, proliferation, migration, invasion, metastasis, apoptosis and resistance to chemotherapy and radiation, as well as influencing overall cancer prognosis. However, exosomal circRNA can function to either promote or inhibit cancer. Exosomes harbouring circRNAs can promote the progression of cancer, the generation of premetastatic niches and the occurrence of metastasis. They can also function in tumour immune regulation and cancer therapy. Due to their unique features and high specificity, the combination of exosomes and circRNAs lends favour to their potential clinical application as cancer diagnostic and prognostic markers.

Notably, although numerous studies have assessed the roles of circRNAs as well as exosomal ncRNAs in cancer, relatively few studies have investigated the functions of exosomal circRNAs. In the near future, scientists worldwide will devote increased attention to exosomal circRNAs research to explore their functions and roles in various diseases, especially in cancer. In addition, cells clear circRNAs via EVs or exosomes, a process that may be used to alleviate circRNA accumulation. Thus, elucidating the mechanisms underlying circRNA clearance and release via exosomes still requires further investigation.

The ultimate goal of medical scientific research is to contribute to improved clinical outcomes for patients. Although studies have already demonstrated the potential functions of circRNAs in cancer diagnosis and therapy, their use as targets in clinical applications, appears to be a promising area of future research, particularly due to the low risk of adverse effects. The use of exosomes as natural drug vectors for the delivery of pharmaceutical cargo and their use in the development of targeted therapeutics should be further studied to enhance delivery efficiency. It is hypothesized that the continued efforts of researchers worldwide will result in therapies involving the delivery of specific circRNAs via exosomes in the near future.

\section{Acknowledgements}

Not applicable.

\section{Funding}

The present study was financially supported by grants from the government-funded Provincial Clinical Medicine Talent Programs in 2017 (grant no. 3010601198073), the Science and Technology Capacity Improvement Projects of Hebei University of Chinese Medicine in 2019 (grant no. KTZ2019019), the Outstanding Student Scientific Research Ability Improvement Projects of Hebei University of Chinese Medicine in 2019 (grant no. YXZ2019002) and the Graduate Innovative Ability Training Projects of Hebei Province in 2020 (grant nos XCXZZBS2020002 and hbu2020bs003).

\section{Availability of data and materials}

Not applicable.

\section{Authors' contributions}

$\mathrm{XG}$ and $\mathrm{XL}$ were the major contributors in the writing and revision of the manuscript. YZ made substantial contributions to the conception or design of the work. QL revised this article critically for important intellectual content. YG analysed the data. CF gave the final approval of the version to be published. HW gave approval for the final version of the manuscript. All authors read and approved the final manuscript. 


\section{Ethics approval and consent to participate}

Not applicable.

\section{Patient consent for publication}

Not applicable.

\section{Competing interests}

The authors declare that they have no competing interests.

\section{References}

1. van Niel G, D'Angelo G and Raposo G: Shedding light on the cell biology of extracellular vesicles. Nat Rev Mol Cell Biol 19: 213-228, 2018

2. Thery C, Zitvogel L and Amigorena S: Exosomes: Composition, biogenesis and function. Nat Rev Immunol 2: 569-579, 2002.

3. Trajkovic K, Hsu C, Chiantia S, Rajendran L, Wenzel D, Wieland F, Schwille P, Brugger B and Simons M: Ceramide triggers budding of exosome vesicles into multivesicular endosomes. Science 319: 1244-1247, 2008

4. Huang K, Fang C, Yi K, Liu X, Qi H, Tan Y, Zhou J, Li Y, Liu M Zhang Y, et al: The role of PTRF/Cavin1 as a biomarker in both glioma and serum exosomes. Theranostics 8: 1540-1557, 2018.

5. Wei Z, Batagov AO, Schinelli S, Wang J, Wang Y, El Fatimy R, Rabinovsky R, Balaj L, Chen CC, Hochberg F, et al: Coding and noncoding landscape of extracellular RNA released by human glioma stem cells. Nat Commun 8: 1145, 2017.

6. Li Y, Zheng Q, Bao C, Li S, Guo W, Zhao J, Chen D, Gu J, He X and Huang S: Circular RNA is enriched and stable in exosomes: A promising biomarker for cancer diagnosis. Cell Res 25 981-984, 2015.

7. Zeng Z, Li Y, Pan Y, Lan X, Song F, Sun J, Zhou K, Liu X, Ren X, Wang F, et al: Cancer-derived exosomal miR-25-3p promotes pre-metastatic niche formation by inducing vascular permeability and angiogenesis. Nat Commun 9: 5395, 2018.

8. Zhang $\mathrm{H}$, Zhu L, Bai M, Liu Y, Zhan Y, Deng T, Yang $H$, Sun W, Wang X, Zhu K, et al: Exosomal circRNA derived from gastric tumor promotes white adipose browning by targeting the miR-133/PRDM16 pathway. Int J Cancer 144: 2501-2515, 2019.

9. Li L, Li C, Wang S, Wang Z, Jiang J, Wang W, Li X, Chen J, Liu K, Li C and Zhu G: Exosomes derived from hypoxic oral squamous cell carcinoma cells deliver miR-21 to normoxic cells to elicit a prometastatic phenotype. Cancer Res 76: 1770-1780, 2016.

10. Silachev DN, Goryunov KV, Shpilyuk MA, Beznoschenko OS, Morozova NY, Kraevaya EE, Popkov VA, Pevzner IB, Zorova LD Evtushenko EA, et al: Effect of MSCs and MSC-derived extracellular vesicles on human blood coagulation. Cells 8: E258, 2019.

11. Shabbir A, Cox A, Rodriguez-Menocal L, Salgado M and Van Badiavas E: Mesenchymal stem cell exosomes induce proliferation and migration of normal and chronic wound fibroblasts, and enhance angiogenesis in vitro. Stem Cells Dev 24: 1635-1647, 2015.

12. Chen G, Huang AC, Zhang W, Zhang G, Wu M, Xu W, Yu Z, Yang J, Wang B, Sun H, et al: Exosomal PD-L1 contributes to immunosuppression and is associated with anti-PD-1 response. Nature 560: 382-386, 2018

13. Kamerkar S, LeBleu VS, Sugimoto H, Yang S, Ruivo CF Melo SA, Lee JJ and Kalluri R: Exosomes facilitate therapeutic targeting of oncogenic KRAS in pancreatic cancer. Nature 546 498-503, 2017

14. Tian T, Zhang HX, He CP, Fan S, Zhu YL, Qi C, Huang NP, Xiao ZD, Lu ZH, Tannous BA and Gao J: Surface functionalized exosomes as targeted drug delivery vehicles for cerebral ischemia therapy. Biomaterials 150: 137-149, 2018.

15. Pan Y, Hui X, Hoo RLC, Ye D, Chan CYC, Feng T, Wang Y, Lam KSL and $\mathrm{Xu} A$ : Adipocyte-secreted exosomal microRNA-34a inhibits M2 macrophage polarization to promote obesity-induced adipose inflammation. J Clin Invest 129 834-849, 2019.

16. Chen LL and Yang L: Regulation of circRNA biogenesis. RNA Biol 12: 381-388, 2015.
17. Cocquerelle C, Mascrez B, Hetuin D and Bailleul B: Mis-splicing yields circular RNA molecules. FASEB J 7: 155-160, 1993.

18. Han B, Chao J and Yao H: Circular RNA and its mechanisms in disease: From the bench to the clinic. Pharmacol Ther 187: 31-44, 2018

19. Vo JN, Cieslik M, Zhang Y, Shukla S, Xiao L, Zhang Y, Wu YM, Dhanasekaran SM, Engelke CG, Cao X, et al: The landscape of circular RNA in cancer. Cell 176: 869-881.e13, 2019.

20. Rybak-Wolf A, Stottmeister C, Glazar P, Jens M, Pino N, Giusti S, Hanan M, Behm M, Bartok O, Ashwal-Fluss R, et al: Circular RNAs in the mammalian brain are highly abundant, conserved, and dynamically expressed. Mol Cell 58: 870-885, 2015.

21. Cheng X, Zhang L, Zhang K, Zhang G, Hu Y, Sun X, Zhao C, Li H, Li YM and Zhao J: Circular RNA VMA21 protects against intervertebral disc degeneration through targeting miR-200c and X linked inhibitor-of-apoptosis protein. Ann Rheum Dis 77: 770-779, 2018.

22. Zheng Q, Bao C, Guo W, Li S, Chen J, Chen B, Luo Y, Lyu D, Li Y, Shi G, et al: Circular RNA profiling reveals an abundant circHIPK3 that regulates cell growth by sponging multiple miRNAs. Nat Commun 7: 11215, 2016.

23. Du WW, Yang W, Liu E, Yang Z, Dhaliwal P and Yang BB: Foxo3 circular RNA retards cell cycle progression via forming ternary complexes with p21 and CDK2. Nucleic Acids Res 44: 2846-2858, 2016.

24. Guarnerio J, Zhang Y, Cheloni G, Panella R, Mae Katon J, Simpson M, Matsumoto A, Papa A, Loretelli C, Petri A, et al: Intragenic antagonistic roles of protein and circRNA in tumorigenesis. Cell Res 29: 628-640, 2019.

25. Zhang PF, Wei CY, Huang XY, Peng R, Yang X, Lu JC, Zhang C, Gao C, Cai JB, Gao PT, et al: Circular RNA circTRIM33-12 acts as the sponge of MicroRNA-191 to suppress hepatocellular carcinoma progression. Mol Cancer 18: 105, 2019.

26. Dong W, Bi J, Liu H, Yan D, He Q, Zhou Q, Wang Q, Xie R, $\mathrm{Su}$ Y, Yang M, et al: Circular RNA ACVR2A suppresses bladder cancer cells proliferation and metastasis through miR-626/EYA4 axis. Mol Cancer 18: 95, 2019.

27. Huang X, Li Z, Zhang Q, Wang W, Li B, Wang L, Xu Z, Zeng A, Zhang X, Zhang X, et al: Circular RNA AKT3 upregulates PIK3R1 to enhance cisplatin resistance in gastric cancer via miR-198 suppression. Mol Cancer 18: 71, 2019.

28. Zhao M, Xu J, Zhong S, Liu Y, Xiao H, Geng L and Liu H: Expression profiles and potential functions of circular RNAs in extracellular vesicles isolated from radioresistant glioma cells. Oncol Rep 41: 1893-1900, 2019.

29. Liang WC, Wong CW, Liang PP, Shi M, Cao Y, Rao ST, Tsui SK, Waye MM, Zhang Q, Fu WM and Zhang JF: Translation of the circular RNA circ $\beta$-catenin promotes liver cancer cell growth through activation of the Wnt pathway. Genome Biol 20: 84, 2019.

30. Wang G, Liu W, Zou Y, Wang G, Deng Y, Luo J, Zhang Y, Li H, Zhang Q, Yang $Y$ and Chen G: Three isoforms of exosomal circPTGR1 promote hepatocellular carcinoma metastasis via the miR449a-MET pathway. EBioMedicine 40: 432-445, 2019.

31. Lu J, Wang YH, Yoon C, Huang XY, Xu Y, Xie JW, Wang JB, Lin JX, Chen QY, Cao LL, et al: Circular RNA circ-RanGAP1 regulates VEGFA expression by targeting miR-877-3p to facilitate gastric cancer invasion and metastasis. Cancer Lett 471 : 38-48, 2020

32. Li Z, Yanfang W, Li J, Jiang P, Peng T, Chen K, Zhao X, Zhang Y, Zhen P, Zhu J and Li X: Tumor-released exosomal circular RNA PDE8A promotes invasive growth via the miR-338/MACC1/MET pathway in pancreatic cancer. Cancer Lett 432: 237-250, 2018.

33. Zhang H, Deng T, Ge S, Liu Y, Bai M, Zhu K, Fan Q, Li J, Ning T, Tian F, et al: Exosome circRNA secreted from adipocytes promotes the growth of hepatocellular carcinoma by targeting deubiquitination-related USP7. Oncogene 38: 2844-2859, 2019.

34. Li J, Li Z, Jiang P, Peng M, Zhang X, Chen K, Liu H, Bi H, Liu X and Li X: Circular RNA IARS (circ-IARS) secreted by pancreatic cancer cells and located within exosomes regulates endothelial monolayer permeability to promote tumor metastasis. J Exp Clin Cancer Res 37: 177, 2018.

35. Bai H, Lei K, Huang F, Jiang Z and Zhou X: Exo-circRNAs: A new paradigm for anticancer therapy. Mol Cancer 18: 56, 2019.

36. $\mathrm{Xu} \mathrm{Z}, \mathrm{Li} \mathrm{P}$, Fan $\mathrm{L}$ and $\mathrm{Wu} \mathrm{M}$ : The potential role of circRNA in tumor immunity regulation and immunotherapy. Front Immunol 9: 9, 2018.

37. Jeck WR and Sharpless NE: Detecting and characterizing circular RNAs. Nat Biotechnol 32: 453-461, 2014. 
38. Memczak S, Jens M, Elefsinioti A, Torti F, Krueger J, Rybak A, Maier L, Mackowiak SD, Gregersen LH, Munschauer M, et al: Circular RNAs are a large class of animal RNAs with regulatory potency. Nature 495: 333-338, 2013.

39. Ashwal-Fluss R, Meyer M, Pamudurti NR, Ivanov A, Bartok O, Hanan M, Evantal N, Memczak S, Rajewsky N and Kadener S: circRNA biogenesis competes with pre-mRNA splicing. Mol Cell 56: 55-66, 2014.

40. Conn SJ, Pillman KA, Toubia J, Conn VM, Salmanidis M, Phillips CA, Roslan S, Schreiber AW, Gregory PA and Goodall GJ: The RNA binding protein quaking regulates formation of circRNAs. Cell 160: 1125-1134, 2015.

41. Ivanov A, Memczak S, Wyler E, Torti F, Porath HT, Orejuela MR, Piechotta M, Levanon EY, Landthaler M, Dieterich C and Rajewsky N: Analysis of intron sequences reveals hallmarks of circular RNA biogenesis in animals. Cell Rep 10: 170-177, 2015.

42. Aktas T, AvsarIlikI,MaticzkaD,Bhardwaj V,Pessoa Rodrigues C, Mittler G, Manke T, Backofen R and Akhtar A: DHX9 suppresses RNA processing defects originating from the Alu invasion of the human genome. Nature 544: 115-119, 2017

43. Zhang XO, Wang HB, Zhang Y, Lu X, Chen LL and Yang L: Complementary sequence-mediated exon circularization. Cell 159: 134-147, 2014.

44. Barrett SP, Wang PL and Salzman J: Circular RNA biogenesis can proceed through an exon-containing lariat precursor. Elife 4: e07540, 2015.

45. Li Z, Huang C, Bao C, Chen L, Lin M, Wang X, Zhong G, Yu B, $\mathrm{Hu}$ W, Dai L, et al: Exon-intron circular RNAs regulate transcription in the nucleus. Nat Struct Mol Biol 22: 256-264, 2015.

46. Zhang Y, Zhang XO, Chen T, Xiang JF, Yin QF, Xing YH, Zhu S, Yang L and Chen LL: Circular intronic long noncoding RNAs. Mol Cell 51: 792-806, 2013.

47. Noto JJ, Schmidt CA and Matera AG: Engineering and expressing circular RNAs via tRNA splicing. RNA Biol 14: 978-984, 2017.

48. Hansen TB, Jensen TI, Clausen BH, Bramsen JB, Finsen B, Damgaard CK and Kjems J: Natural RNA circles function as efficient microRNA sponges. Nature 495: 384-388, 2013.

49. Lu Y,Deng X,Xiao G,Zheng X,MaL and Huang W: Circ 0001730 promotes proliferation and invasion via the miR-326/Wnt7B axis in glioma cells. Epigenomics 11: 1335-1352, 2019.

50. Abdelmohsen K, Panda AC, Munk R, Grammatikakis I, Dudekula DB, De S, Kim J, Noh JH, Kim KM, Martindale JL and Gorospe M: Identification of HuR target circular RNAs uncovers suppression of PABPN1 translation by CircPABPN1. RNA Biol 14: 361-369, 2017.

51. Kong P, Yu Y, Wang L, Dou YQ, Zhang XH, Cui Y, Wang HY, Yong YT, Liu YB, Hu HJ, et al: Circ-Sirt1 controls NF-кB activation via sequence-specific interaction and enhancement of SIRT1 expression by binding to miR-132/212 in vascular smooth muscle cells. Nucleic Acids Res 47: 3580-3593, 2019.

52. Zhang M, Huang N, Yang X, Luo J, Yan S, Xiao F, Chen W, Gao X, Zhao K, Zhou H, et al: A novel protein encoded by the circular form of the SHPRH gene suppresses glioma tumorigenesis. Oncogene 37: 1805-1814, 2018.

53. Yang $Y$, Fan X, Mao M, Song X, Wu P, Zhang Y, Jin Y, Yang Y, Chen LL, Wang Y, et al: Extensive translation of circular RNAs driven by $\mathrm{N}^{6}$-methyladenosine. Cell Res 27: 626-641, 2017.

54. Valadi H, Ekstrom K, Bossios A, Sjostrand M, Lee JJ and Lotvall JO: Exosome-mediated transfer of mRNAs and microRNAs is a novel mechanism of genetic exchange between cells. Nat Cell Biol 9: 654-659, 2007.

55. Yin Y, Cai X, Chen X, Liang H, Zhang Y, Li J, Wang Z, Chen X, Zhang W, Yokoyama S, et al: Tumor-secreted miR-214 induces regulatory T cells: A major link between immune evasion and tumor growth. Cell Res 24: 1164-1180, 2014

56. Yin J, Zeng A, Zhang Z, Shi Z, Yan W and You Y: Exosomal transfer of miR-1238 contributes to temozolomide-resistance in glioblastoma. EBioMedicine 42: 238-251, 2019.

57. Shao N, Xue L, Wang R, Luo K, Zhi F and Lan Q: MiR-454-3p is an exosomal biomarker and functions as a tumor suppressor in glioma. Mol Cancer Ther 18: 459-469, 2019.

58. Qu L, Ding J, Chen C, Wu ZJ, Liu B, Gao Y, Chen W, Liu F, Sun W, Li XF, et al: Exosome-transmitted lncARSR promotes sunitinib resistance in renal cancer by acting as a competing endogenous RNA. Cancer Cell 29: 653-668, 2016

59. Dou Y, Cha DJ, Franklin JL, Higginbotham JN, Jeppesen DK, Weaver AM, Prasad N, Levy S, Coffey RJ, Patton JG and Zhang B: Circular RNAs are down-regulated in KRAS mutant colon cancer cells and can be transferred to exosomes. Sci Rep 6: 37982,2016
60. Liang D, Tatomer DC, Luo Z, Wu H, Yang L, Chen LL, Cherry S and Wilusz JE: The output of protein-coding genes shifts to circular RNAs when the pre-mRNA processing machinery is limiting. Mol Cell 68: 940-954.e3, 2017.

61. Jeck WR, Sorrentino JA, Wang K, Slevin MK, Burd CE, Liu J, Marzluff WF and Sharpless NE: Circular RNAs are abundant, conserved, and associated with ALU repeats. RNA 19: 141-157, 2013.

62. Zhang Y, Xue W, Li X, Zhang J, Chen S, Zhang JL, Yang L and Chen LL: The biogenesis of nascent circular RNAs. Cell Rep 15: 611-624, 2016.

63. Lasda E and Parker R: Circular RNAs co-precipitate with extracellular vesicles: A possible mechanism for circRNA clearance. PLoS One 11: e0148407, 2016.

64. Hong BS, Cho JH, Kim H, Choi EJ, Rho S, Kim J, Kim JH, Choi DS, Kim YK, Hwang D and Gho YS: Colorectal cancer cell-derived microvesicles are enriched in cell cycle-related mRNAs that promote proliferation of endothelial cells. BMC Genomics 10: 556, 2009.

65. He C, Zheng S, Luo Y and Wang B: Exosome theranostics: Biology and translational medicine. Theranostics 8: 237-255, 2018.

66. Raposo G and Stoorvogel W: Extracellular vesicles: Exosomes, microvesicles, and friends. J Cell Biol 200: 373-383, 2013.

67. Perez-Boza J, Lion M and Struman I: Exploring the RNA landscape of endothelial exosomes. RNA 24: 423-435, 2018.

68. Santangelo L, Giurato G, Cicchini C, Montaldo C, Mancone C, Tarallo R, Battistelli C, Alonzi T, Weisz A and Tripodi M: The RNA-binding protein SYNCRIP is a component of the hepatocyte exosomal machinery controlling microRNA sorting. Cell Rep 17: 799-808, 2016.

69. Shurtleff MJ, Temoche-Diaz MM, Karfilis KV, Ri S and Schekman R: Y-box protein 1 is required to sort microRNAs into exosomes in cells and in a cell-free reaction. Elife 5: e19276, 2016.

70. Villarroya-Beltri C, Gutierrez-Vazquez C, Sanchez-Cabo F, Perez-Hernandez D, Vazquez J, Martin-Cofreces N, Martinez-Herrera DJ, Pascual-Montano A, Mittelbrunn M and Sanchez-Madrid F: Sumoylated hnRNPA2B1 controls the sorting of miRNAs into exosomes through binding to specific motifs. Nat Commun 4: 2980, 2013.

71. Scilla KA and Rolfo C: The role of circulating tumor DNA in lung cancer: Mutational analysis, diagnosis, and surveillance now and into the future. Curr Treat Options Oncol 20: $61,2019$.

72. Caby MP, Lankar D, Vincendeau-Scherrer C, Raposo G and Bonnerot C: Exosomal-like vesicles are present in human blood plasma. Int Immunol 17: 879-887, 2005

73. Qin W, Tsukasaki Y, Dasgupta S, Mukhopadhyay N, Ikebe M and Sauter ER: Exosomes in human breast milk promote EMT. Clin Cancer Res 22: 4517-4524, 2016.

74. Katsiougiannis S, Chia D, Kim Y, Singh RP and Wong DT: Saliva exosomes from pancreatic tumor-bearing mice modulate NK cell phenotype and antitumor cytotoxicity. FASEB J 31: 998-1010, 2017.

75. McKiernan J, Donovan MJ, O'Neill V, Bentink S, Noerholm M, Belzer S, Skog J, Kattan MW, Partin A, Andriole G, et al: A novel urine exosome gene expression assay to predict high-grade prostate cancer at initial biopsy. JAMA Oncol 2: $882-889,2016$

76. Barcelo M, Castells M, Bassas L, Vigues F and Larriba S: Semen miRNAs contained in exosomes as non-invasive biomarkers for prostate cancer diagnosis. Sci Rep 9: 13772, 2019.

77. Kong FL, Wang XP, Li YN and Wang HX: The role of exosomes derived from cerebrospinal fluid of spinal cord injury in neuron proliferation in vitro. Artif Cells Nanomed Biotechnol 46: 200-205, 2018

78. Dixon CL, Sheller-Miller S, Saade GR, Fortunato SJ, Lai A Palma C, Guanzon D, Salomon C and Menon R: Amniotic fluid exosome proteomic profile exhibits unique pathways of term and preterm labor. Endocrinology 159: 2229-2240, 2018.

79. Levanen B, Bhakta NR, Torregrosa Paredes P, Barbeau R, Hiltbrunner S, Pollack JL, Skold CM, Svartengren M, Grunewald J, Gabrielsson S, et al: Altered microRNA profiles in bronchoalveolar lavage fluid exosomes in asthmatic patients. J Allergy Clin Immunol 131: 894-903, 2013.

80. Wang L, Shen C, Wang Y, Zou T, Zhu H, Lu X, Li L, Yang B, Chen J, Chen S, et al: Identification of circular RNA Hsa circ_0001879 and Hsa_circ_0004104 as novel biomarkers for coronary artery disease. Atherosclerosis 286: 88-96, 2019. 
81. Zhang Y, Yu F, Bao S and Sun J: Systematic characterization of circular RNA-associated ceRNA network identified novel circRNA biomarkers in Alzheimer's disease. Front Bioeng Biotechnol 7: 222, 2019.

82. Li H, Li K, Lai W, Li X, Wang H, Yang J, Chu S, Wang H, Kang C and Qiu Y: Comprehensive circular RNA profiles in plasma reveals that circular RNAs can be used as novel biomarkers for systemic lupus erythematosus. Clin Chim Acta 480: 17-25, 2018.

83. Dai X, Chen C, Yang Q, Xue J, Chen X, Sun B, Luo F, Liu X, Xiao T, Xu H, et al: Exosomal circRNA_100284 from arsenite-transformed cells, via microRNA-217 regulation of EZH2, is involved in the malignant transformation of human hepatic cells by accelerating the cell cycle and promoting cell proliferation. Cell Death Dis 9: 454, 2018.

84. Li Y, Zhao J, Yu S, Wang Z, He X, Su Y, Guo T, Sheng H, Chen J, Zheng Q, et al: Extracellular vesicles long RNA sequencing reveals abundant mRNA, circRNA, and lncRNA in human blood as potential biomarkers for cancer diagnosis. Clin Chem 65: 798-808, 2019

85. Chen J, Chen T, Zhu Y,Li Y,Zhang Y,Wang Y,LiX, Xie X, Wang J, Huang M, et al: CircPTN sponges miR-145-5p/miR-330-5p to promote proliferation and stemness in glioma. J Exp Clin Cancer Res 38: 398, 2019.

86. Meng Q, Li S, Liu Y, Zhang S, Jin J, Zhang Y, Guo C, Liu B and Sun Y: Circular RNA circSCAF11 accelerates the glioma tumorigenesis through the miR-421/SP1/VEGFA Axis. Mol Ther Nucleic Acids 17: 669-677, 2019.

87. Ma P, Pan Y, Li W, Sun C, Liu J, Xu T and Shu Y: Extracellular vesicles-mediated noncoding RNAs transfer in cancer. J Hematol Oncol 10: 57, 2017.

88. Wang LP, Peng XY, Lv XQ, Liu L, Li XL, He X, Lv F, Pan Y, Wang L, Liu KF and Zhang XM: High throughput circRNAs sequencing profile of follicle fluid exosomes of polycystic ovary syndrome patients. J Cell Physiol: Feb 18, 2019 (Epub ahead of print).

89. Dunne RF, Loh KP, Williams GR, Jatoi A, Mustian KM and Mohile SG: Cachexia and sarcopenia in older adults with cancer: A comprehensive review. Cancers (Basel) 11: E1861, 2019.

90. Su Y, Lv X, Yin W, Zhou L, Hu Y, Zhou A and Qi F: circRNA Cdrlas functions as a competitive endogenous RNA to promote hepatocellular carcinoma progression. Aging (Albany NY) 11: 8182-8203, 2019.

91. Guan X, Zong ZH, Liu Y, Chen S, Wang LL and Zhao Y: CircPUM1 promotes tumorigenesis and progression of ovarian cancer by sponging miR-615-5p and miR-6753-5p. Mol Ther Nucleic Acids 18: 882-892, 2019.

92.Kim KM, Abdelmohsen K, Mustapic M, Kapogiannis D and Gorospe M: RNA in extracellular vesicles. Wiley Interdiscip Rev RNA 8: 2017

93. Hu C, Chen M, Jiang R, Guo Y, Wu M and Zhang X: Exosome-related tumor microenvironment. J Cancer 9: 3084-3092, 2018.

94. Bebelman MP, Smit MJ, Pegtel DM and Baglio SR: Biogenesis and function of extracellular vesicles in cancer. Pharmacol Ther 188: 1-11, 2018.

95. Zhang X, Wang S, Wang H, Cao J, Huang X, Chen Z, Xu P, Sun G, Xu J, Lv J and Xu Z: Circular RNA circNRIP1 acts as a microRNA-149-5p sponge to promote gastric cancer progression via the AKT1/mTOR pathway. Mol Cancer 18: 20, 2019.

96. Chen X, Chen RX, Wei WS, Li YH, Feng ZH, Tan L, Chen JW, Yuan GJ, Chen SL, Guo SJ, et al: PRMT5 circular RNA promotes metastasis of urothelial carcinoma of the bladder through sponging miR-30c to induce epithelial-mesenchymal transition. Clin Cancer Res 24: 6319-6330, 2018.

97. LeBleu VS and Kalluri R: Exosomes exercise inhibition of anti-tumor immunity during chemotherapy. Immunity 50 : 547-549, 2019.

98. Lema DA and Burlingham WJ: Role of exosomes in tumour and transplant immune regulation. Scand J Immunol 90: e12807, 2019

99. Wolfers J, Lozier A, Raposo G, Regnault A, Thery C, Masurier C, Flament C, Pouzieux S, Faure F, Tursz T, et al: Tumor-derived exosomes are a source of shared tumor rejection antigens for CTL cross-priming. Nat Med 7: 297-303, 2001

100. Xie Y, Dang W, Zhang S, Yue W, Yang L, Zhai X, Yan Q and $\mathrm{Lu} \mathrm{J}$ : The role of exosomal noncoding RNAs in cancer. Mol Cancer 18: 37, 2019.

101. Mrizak D, Martin N, Barjon C, Jimenez-Pailhes AS, Mustapha R, Niki T, Guigay J, Pancre V, de Launoit Y, Busson P, et al: Effect of nasopharyngeal carcinoma-derived exosomes on human regulatory T cells. J Natl Cancer Inst 107: 363, 2015.
102. Yamada N, Kuranaga Y, Kumazaki M, Shinohara H, Taniguchi K and Akao Y: Colorectal cancer cell-derived extracellular vesicles induce phenotypic alteration of $\mathrm{T}$ cells into tumor-growth supporting cells with transforming growth factor- $\beta 1$-mediated suppression. Oncotarget 7: 27033-27043, 2016

103. Li P, Liu C, Yu Z and Wu M: New insights into regulatory T cells: Exosome- and non-coding RNA-mediated regulation of homeostasis and resident treg cells. Front Immunol 7: 574 , 2016.

104. Que RS, Lin C, Ding GP, Wu ZR and Cao LP: Increasing the immune activity of exosomes: The effect of miRNA-depleted exosome proteins on activating dendritic cell/cytokine-induced killer cells against pancreatic cancer. J Zhejiang Univ Sci B 17: 352-360, 2016

105. Dudda JC, Salaun B, Ji Y, Palmer DC, Monnot GC, Merck E, Boudousquie C, Utzschneider DT, Escobar TM, Perret R, et al: MicroRNA-155 is required for effector CD8+ $\mathrm{T}$ cell responses to virus infection and cancer. Immunity 38 : 742-753, 2013

106. Morishita M, Takahashi Y, Matsumoto A, Nishikawa M and Takakura Y: Exosome-based tumor antigens-adjuvant co-delivery utilizing genetically engineered tumor cell-derived exosomes with immunostimulatory CpG DNA. Biomaterials 111: $55-65,2016$

107. Kim MS, Haney MJ, Zhao Y, Yuan D, Deygen I, Klyachko NL, Kabanov AV and Batrakova EV: Engineering macrophage-derived exosomes for targeted paclitaxel delivery to pulmonary metastases: In vitro and in vivo evaluations. Nanomedicine 14: 195-204, 2018

108. Aqil F, Munagala R, Jeyabalan J, Agrawal AK, Kyakulaga AH, Wilcher SA and Gupta RC: Milk exosomes-natural nanoparticles for siRNA delivery. Cancer Lett 449: 186-195, 2019.

109. Usman WM, Pham TC, Kwok YY, Vu LT, Ma V, Peng B, Chan YS, Wei L, Chin SM, Azad A, et al: Efficient RNA drug delivery using red blood cell extracellular vesicles. Nat Commun 9: 2359, 2018

110. Roma-Rodrigues C, Raposo LR, Cabral R, Paradinha F, Baptista PV and Fernandes AR: Tumor microenvironment modulation via gold nanoparticles targeting malicious exosomes: Implications for cancer diagnostics and therapy. Int J Mol Sci 18: E162, 2017.

111. Kim SM, Yang Y, Oh SJ, Hong Y, Seo M and Jang M: Cancer-derived exosomes as a delivery platform of CRISPR/Cas9 confer cancer cell tropism-dependent targeting. J Control Release 266: 8-16, 2017.

112. Qi H, Liu C, Long L, Ren Y, Zhang S, Chang X, Qian X, Jia H, Zhao J, Sun J, et al: Blood exosomes endowed with magnetic and targeting properties for cancer therapy. ACS Nano 10: 3323-3333, 2016.

113. Agrawal AK, Aqil F, Jeyabalan J, Spencer WA, Beck J, Gachuki BW, Alhakeem SS, Oben K, Munagala R, Bondada S and Gupta RC: Milk-derived exosomes for oral delivery of paclitaxel. Nanomedicine 13: 1627-1636, 2017.

114. O'Loughlin AJ,Mager I, de Jong OG, Varela MA, Schiffelers RM, El Andaloussi S, Wood MJA and Vader P: Functional delivery of lipid-conjugated siRNA by extracellular vesicles. Mol Ther 25 : 1580-1587, 2017

115. Lunavat TR, Jang SC, Nilsson L, Park HT, Repiska G, Lasser C, Nilsson JA, Gho YS and Lotvall J: RNAi delivery by exosome-mimetic nanovesicles - Implications for targeting c-Myc in cancer. Biomaterials 102: 231-238, 2016.

116. Jackson AL, Burchard J, Leake D, Reynolds A, Schelter J, Guo J, Johnson JM, Lim L, Karpilow J, Nichols K, et al: Position-specific chemical modification of siRNAs reduces 'off-target' transcript silencing. RNA 12: 1197-1205, 2006.

117. Wesselhoeft RA, Kowalski PS and Anderson DG: Engineering circular RNA for potent and stable translation in eukaryotic cells. Nat Commun 9: 2629, 2018.

118. Su H, Tao T, Yang Z, Kang X, Zhang X, Kang D, Wu S and Li C: Circular RNA cTFRC acts as the sponge of microRNA-107 to promote bladder carcinoma progression. Mol Cancer 18: 27, 2019.

119. Chen S, Huang V, Xu X, Livingstone J, Soares F, Jeon J, Zeng Y, Hua JT, Petricca J, Guo H, et al: Widespread and functional RNA circularization in localized prostate cancer. Cell 176: 831-843.e22, 2019.

120.Zeng K, Chen X, Xu M, Liu X, Hu X, Xu T, Sun H, Pan Y, He B and Wang S: CircHIPK3 promotes colorectal cancer growth and metastasis by sponging miR-7. Cell Death Dis 9: 417,2018 . 
121. Bai N, Peng E, Qiu X, Lyu N, Zhang Z, Tao Y, Li X and Wang Z: CircFBLIM1 act as a ceRNA to promote hepatocellular cancer progression by sponging miR-346. J Exp Clin Cancer Res 37: $172,2018$.

122. Wang R, Zhang S, Chen X, Li N, Li J, Jia R, Pan Y and Liang H: CircNT5E acts as a sponge of miR-422a to promote glioblastoma tumorigenesis. Cancer Res 78: 4812-4825, 2018

123. Wang R, Zhang S, Chen X, Li N, Li J, Jia R, Pan Y and Liang H: EIF4A3-induced circular RNA MMP9 (circMMP9) acts as a sponge of miR-124 and promotes glioblastoma multiforme cell tumorigenesis. Mol Cancer 17: 166, 2018.

124. Liu T, Zhang X, Du L, Wang Y, Liu X, Tian H, Wang L, Li P, Zhao Y, Duan W, et al: Exosome-transmitted miR-128-3p increase chemosensitivity of oxaliplatin-resistant colorectal cancer. Mol Cancer 18: 43, 2019.

125. Qin X, Guo H, Wang X, Zhu X, Yan M, Wang X, Xu Q, Shi J, Lu E, Chen W and Zhang J: Exosomal miR-196a derived from cancer-associated fibroblasts confers cisplatin resistance in head and neck cancer through targeting CDKN1B and ING5. Genome Biol 20: 12, 2019.
126. Geng X, Jia Y, Zhang Y, Shi L, Li Q, Zang A and Wang H: Circular RNA: Biogenesis, degradation, functions and potential roles in mediating resistance to anticarcinogens. Epigenomics 12: 267-283, 2020.

127. Zhao Z, Ji M, Wang Q, He N and Li Y: Circular RNA Cdrlas Upregulates SCAI to suppress cisplatin resistance in ovarian cancer via miR-1270 suppression. Mol Ther Nucleic Acids 18: 24-33, 2019.

128. Liu F, Zhang J, Qin L, Yang Z, Xiong J, Zhang Y, Li R, Li S, Wang H, Yu B, et al: Circular RNA EIF6 (Hsa_circ_0060060) sponges miR-144-3p to promote the cisplatin-resistance of human thyroid carcinoma cells by autophagy regulation. Aging (Albany NY) 10: 3806-3820, 2018.

This work is licensed under a Creative Commons Attribution-NonCommercial-NoDerivatives 4.0 International (CC BY-NC-ND 4.0) License. 